\title{
Polarization of Hard X-Rays in October-November, 2003 Solar Flares Observed Onboard CORONAS-F Satellite
}

\author{
A. V. Bogomolov ${ }^{1} \dagger$, Yu. I. Denisov ${ }^{1}$, Yu. I. Logachev ${ }^{1}$, \\ O. V. Morozov ${ }^{1}$, I. N. Myagkova ${ }^{1}$, S. I. Svertilov ${ }^{1}$, \\ I. A. Zhitnik ${ }^{2}$, A. P. Ignat'ev ${ }^{2}$, S. N. Oparin ${ }^{2}$, and A. A. Pertsov ${ }^{2}$ \\ ${ }^{1}$ Skobeltsyn Institute of Nuclear Physics, Moscow State University, Vorob'evy Gory, Moscow, \\ 119992, Moscow, Russia e-mail: aabboogg@nm.ru. \\ ${ }^{2}$ Lebedev Physical Institute, Russian Academy of Sccience, Leninskii pr., 53, Moscow, 119991, \\ Russia
}

\begin{abstract}
Hard X-ray radiation from several powerful solar flares was detected in OctoberNovember, 2003 by the SPR-N hard X-ray polarimeter onboard CORONAS-F satellite. In the October, 29 solar flare (20:40 - 21:00 UT) the X-ray radiation was strongly polarized (more than $70 \%$ of photons with energies $40-100 \mathrm{keV}$ ). In the flares 28.10 .03 and 04.11 .03 the emission was not polarized. The upper limits for the part of polarized photons at the level of tens percents were obtained in these flares.
\end{abstract}

The possibility of observing the polarization of hard X-ray radiation is of particular importance, because bremsstrahlung polarization suggests the existence of energetic electron beams in the source. The earlier experiments on measuring X-ray polarization of solar flares were made only in soft energy range (Tindo et al. (1971), Tindo et al. (1972), Zhitnik, Tindo \& Urnov (1989)). This work presents the results of studying hard X-ray polarization of the powerful solar flares 28.10.2003, 29.10.2003 and 4.11.2003 in the experiment SPR-N onboard CORONAS-F satellite. All these flares occured in the same active region 10486 .

The SPR-N detector unit includes a patrol detector for monitoring of the Sun in the energy ranges 15-40 and 40-100 keV and a polarization detector system for measuring polarization in the ranges $20-40,40-60$ and $60-100 \mathrm{keV}$. The X-ray radiation falls on the beryllium prism where it suffers Thomson scattering. Six CsI(Na) sensors, located on the faces of the prism detect the direction of photon scattering. When a plane-polarized radiation is recorded, most of the photons are scattered normally to the polarization plane. Non-polarized radiation scatters isotropically. Thus, the relations of sensor count rates give an information about both the part of polarized photons and the location of polarization plane at the Sun. Detailed description of the hard X-ray polarimeter SPR-N and the first results of the experiment SPR-N onboard CORONAS-F satellite (since August, 15, 2001) are presented in (Bogomolov et al. (2003)).

The experimental data, obtained during the 29.10.2003 flare (20:40-21:00 UT) show that the hard X-ray radiation is strongly polarized. Part of polarized photons and the location of polarization plane for this flare are presented on Fig. 1. The maximal part of polarization was observed in two peaks of hard radiation (20:43 and 20:51 UT) observed also by the SPR-N patrol detector and SONG instrument onboard CORONAS-F

$\dagger$ Present address: Skobeltsyn Institute of Nuclear Physics, Moscow State University, Vorob'evy Gory, Moscow, 119992, Russia. 


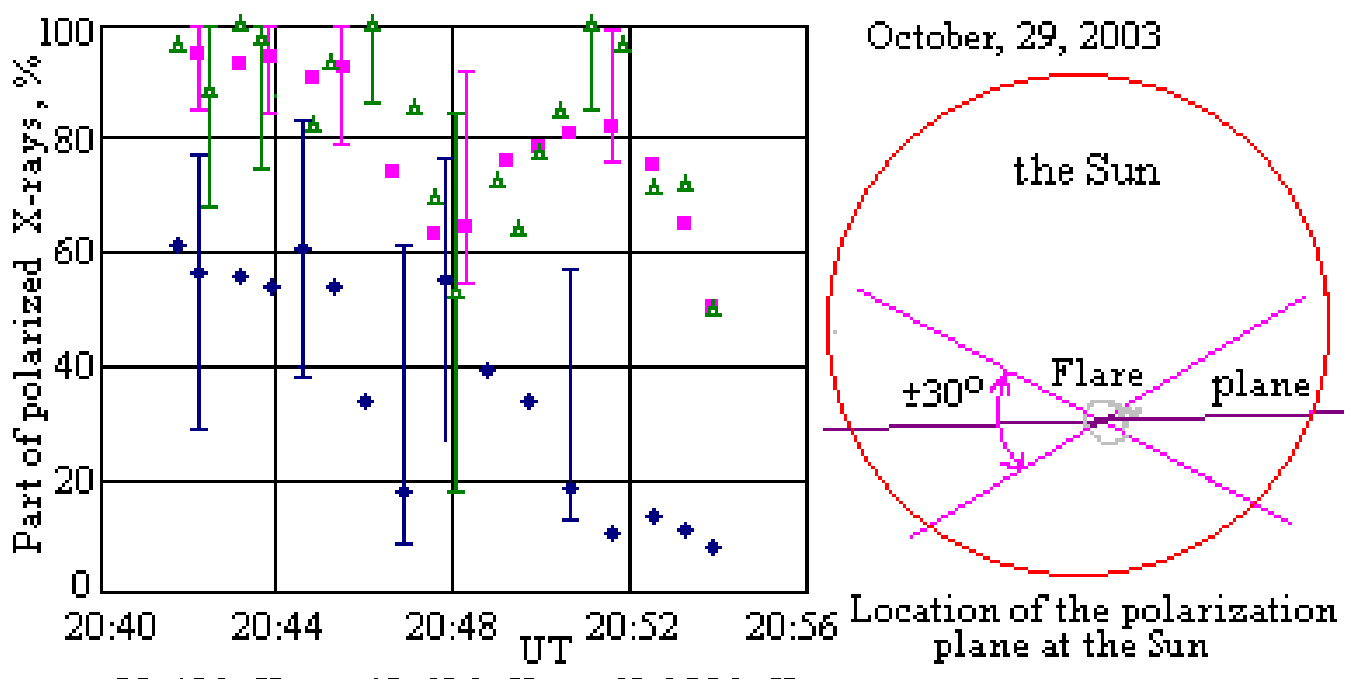

- $20-40 \mathrm{keV}=40-60 \mathrm{keV}$ \& $60-100 \mathrm{keV}$

Figure 1. Part of polarized photons and location of polarization plane in a flare 29.10.2003

(Kuznetsov et al, see this book). In the energy channel 40-60 keV the part of polarized emission is $>85 \%$ in the first peak and $>75 \%$ in the second peak. Similar situation is in the channel $60-100 \mathrm{keV}$. In the softer channel $20-40 \mathrm{keV}$ the X-ray emission was not polarized so strongly (see figure 1 )

In the 28.10.2003 flare (11:00-11:20 UT) and the first stage of 4.11.2003 flare $(19: 34$ UT) the hard X-ray emission was not polarized significantly. Only upper limits $25 \%$ for 28.10 .03 and $40 \%$ for 4.11 .03 were obtained. In the main peak of the flare 4.11 .03 polarization was not estimated because the satellite at this time crossed the radiation belts. 28.10.03 and 29.10.03 errors of measurements are mainly connected with the account of possibble different sensitivity of detector units to a flare radiation. In a flare 4.11.03 statistics plays more important role in an accuracy of measurements.

Before 29.10.2003 flare significant hard X-ray polarization was not observed. In a X5.3 flare 25.08.2001 we obtained, that part of polarized emission was less, than $8.5 \%$.

\section{References}

Bogomolov A. V., Denisov Yu. I., Kuznetsov S. N., et al. 2003 Astron. Vestnik 37, 127-136.

Tindo, I. P., Ivanov, V. P., Mandelstam, S. L., et al. 1971 Kosm. Issled. 9, 116.

Tindo, I., Ivanov, V., Mandelstam, S., et al. 1972 Solar Phys. 24, 429.

Zhitnik I. A., Tindo I. P. \& Urnov A. M. 1989 Trudy FIAN AN SSSR, Moscow 195, 3-18. 